\title{
Ultrastructural Analysis of Black Lipid Membranes
}

\author{
P. H. J. Th. Ververgaert and P. F. Elbers \\ Centrum voor Submicroscopisch Onderzoek \\ van Biologische Objecten der Rijksuniversiteit \\ Utrecht, The Netherlands
}

(Received 27 April 1970, and in revised form 20 November 1970)

\begin{abstract}
Black films of egg phosphatidylcholine were investigated by electron microscopy of thin sections. A stabilizing method was developed based on a tricomplex reaction of the lipid, in order to avoid difficulties of interpretation pertaining to $\mathrm{OsO}_{4}$ and $\mathrm{KMnO}_{4}$-fixation techniques. Membranes were essentially trilaminar, as could be demonstrated by specimen tilting, and extensively undulated.

Densitometer tracings of micrographs revealed a peak to peak width of $45 \AA$. Corrected for electron radiation damage this width would amount to $60 \AA$. Taking into account the thiokness of the polar layers, this would mean a total thickness of $69 \AA$ of the native membrane.
\end{abstract}

\section{Introduction}

Since black lipid membranes display a number of physical and chemical properties that are common to cellular membranes (Tien \& Diana, 1968) their use as experimental models can greatly enhance our knowledge of biological membranes. Structural deviations from biological mombranes can, however, be expected, especially because a large amount of apolar, non-biological material is assumed to be present within the black film (Cook, Redwood, Taylor \& Haydon, 1968).

The thickness of ultrathin films has been calculated from capacitance (Hanai, Haydon \& Taylor, 1964) and optical reflection (Tien, 1968) data, but so far great differences between the results have been introduced by different assumptions about the dielectric constant and refractive index of the film. Nevertheless, the thickness values obtained in these ways roughly match electron microscope measurements of cellular membranes (Tien \& Diana, 1968).

An electron microscope investigation of black lipid films would enable us to compare directly the thickness of black lipid membranes with that of cellular membranes. Moreover, insight could be obtained into small structural variations within the film, and such a study would offer the additional advantage of an independent measurement of polar and apolar moieties of the black film.

Henn, Decker, Greenawalt \& Thompson (1967) could visualize, after $\mathrm{KMnO}_{4}$ fixation, a phosphatidylethanolamine film as a trilaminar structure with a wide variation in thickness (37.5 $\AA$ to $116.0 \AA$ ) interrupted by multilaminar areas. This method, however, worked only when $\mathrm{La}\left(\mathrm{NO}_{3}\right)_{3}$ was present during fixation with $\mathrm{KMnO}_{4}$. The classical fixation methods applied to films of different composition did not yield specimens for electron microscopy. The relevance of their results, however, 
was criticized especially with regard to the extent of black lipid membrane reorganization during fixation with $\mathrm{KMnO}_{4}$ (Andrews \& Haydon, 1968). On the other hand Mueller, Rudin, Tien \& Wescott (1964) using $\mathrm{OsO}_{4}$ fixation of a mixed-lipid film, could not reveal the triple-layered character of the lipid membrane, but showed a micrograph featuring a $90 \AA$ wide, continuous band. In their study, a possible trilaminar character of the black lipid membranes perhaps was obscured by a film orientation not parallel to the electron beam.

The chemical events occuring during $\mathrm{OsO}_{4}$ fixation have been the subject of many investigations (Riemersma \& Booy, 1962; Riemersma, 1968; Stoeckenius \& Mahr, 1965; Korn, 1966a,b). Contrary to the work of Stoeckenius and Riemersma, the experiments by Korn did not lend support to the idea that the osmium deposit is located in the polar part of the membrane. Arguments in favour of this idea can be derived from electron microscopic studies on multilayers of brain lipids (Robertson, 1958) and uranyl linolenate (Stoeckenius, 1960). In a general sense, Elbers (1964) pointed to the vicissitudes of $\mathrm{KMnO}_{4}$ fixation, whereas from X-ray diffraction studies by Moretz, Akers \& Parsons (1969) evidence is put forward that the hydrocarbon part of membrane structures becomes profoundly disturbed by both $\mathrm{OsO}_{4}$ and $\mathrm{KMnO}_{4}$. An indication of such disturbance is found also in the dramatic increase of cell membrane permeability upon the addition of $\mathrm{OsO}_{4}$ to the physiological medium of the cell (Elbers, 1965).

Consequently, the interpretation of electron microscope data would be facilitated if the thin film could be fixed or stabilized right after its formation and in a way to prevent restructuring or demixing. Moreover the method should exclude any doubt on contrast localization in the micrographs. We have met these conditions in a stabilization method based on the work of Elbers, Ververgaert \& Demel (1965). In this so-called tricomplex fixation a reaction is achieved between the zwitterionic group of phosphatidylcholine and a suitable anion and cation.

The attachment of these ions to the phosphorylcholine part of the phospholipid is governed by valency, radius and polarizing power of the ions and also by the polarizability of the phosphate and choline group. In such a tricomplex the effective electro. static attraction is very high, so that a higher resistance of the lipid bilayer against disturbing forces may be expected. The complexes are dehydrated without further fixation, embedded and studied in thin sections without further staining. Sufficient contrast was obtained by choosing heavy-metal containing ions as participants in the tricomplex reaction.

\section{Materials and Methods}

(a) Chemicals

Analytical grade chloroform, tetradecane and $\alpha$-tocopherol were obtained from Merck and used without further purification. Phosphatidylcholine was isolated from egg yolk according to Pangborn (1951). Subsequent chromatography on silica yielded a whitish preparation free from any contamination detectable on thin layer chromatography plates.

\section{(b) Filmholders}

Araldite rings with an outer diameter of $0.2 \mathrm{~cm}$, and an inner diameter of $0.16 \mathrm{~cm}$ were mounted on small Araldite rods. This particular resin was used because it meets three important requirements: insolubility, no swelling in acetone, and good embedding properties in epoxy resin. 
(c) Film solutions and draining process

A drop $(0.04 \mathrm{cc})$ of a $2 \%$ lecithin solution in chloroform-methanol- $\alpha$ tocopherol (or tetradecane) $3: 2: 2$, was applied with a microsyringe in the ring submerged in a salt solution. Subsequently a small amount of the lipid solution was sucked away until interference colours became visible. Black spots appeared in most instances spontaneously within $10 \mathrm{~min}$. Thinning to black could also be triggored by touching the film with a fino needle.

Draining of the film proceeded at $41^{\circ} \mathrm{C}$ in either $10^{-4} \mathrm{~N}-\mathrm{UO}_{2}\left(\mathrm{NO}_{3}\right)_{2}$ or $10^{-2} \mathrm{~N}-\mathrm{Co}\left(\mathrm{NO}_{3}\right)_{2}$. Afterwards KCNS or ammonium molybdate was added giving a $10^{-2} \mathrm{~N}$ end concentration. Best results were obtained with $\mathrm{UO}_{2}\left(\mathrm{NO}_{3}\right)_{2}$ and KCNS. Underneath the surface of this salt solution the rings were transported to a gelatine capsule containing molten $2 \%$ agar. The gelatine capsule was previously hardened by glutaraldehyde and could be removed easily before dehydration.

\section{(d) Electron microscopy}

Blooks were dehydrated in acetone at $5^{\circ} \mathrm{C}$, in order to avoid loss of lipid, and embodded in Araldite. During trimming of the embedded specimens the location of the film could be observed as a very faint line of irregularities produced by the rough edge of a razor blade. A smonth surface, however, without any indication of the bilayer was seen after sectioning with a glass knife. Inside the block no trace of the lipid film could be observed.

Thin sections were cut on a Reichert ultramicrotome, collected on parlodion-covered brass rings with a 1-mm hole and, without further staining, studied with a Siemens IA or a Philips EM200 electron microscope.

\section{(e) Quantitative electron microscopy}

Thickness measurements were performed with a Siemens Elmiskop IA at an initial magnification in the 56000 $\times$ range as described by Elbers \& Pieters (1964). Tilting of the specimen was carried out in a Philips EM300 electron microscope equipped with a gonio. meter stage and a rotation holder.

Density distribution across the bilayer was recorded from the micrographs with a scanning densitometer.

\section{Results}

Black lipid membranes are visualized at low magnifications on electron micrographs as a continuous dark line interrupted by thicker, 100 to $150 \AA$ wide, lens-shaped structures (Plate I, top). At sufficiently high magnifications trilaminar structures can be detected (Plate I, middle). Single dark parts are observed as well, but by tilting the specimen over $10^{\circ}$ we could prove that these result from the geometrical projection of a trilaminar structure not parallel to the electron beam in the original specimen orientation (Plate I, bottom). Measurements were made on photographic plates according to Figure 1. In this Figure, theoretical densitometer curves are given for three orientations of the sectioned black lipid membranes with respect to the electron beam.
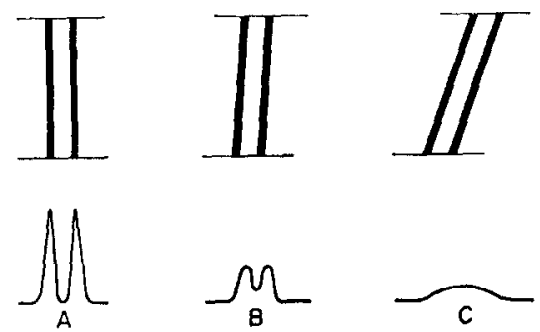

Fia. 1. Three different film orientations within a section and corresponding theoretical densitometer curves. The black lipid membrane sections are drawn to scale. Only densitometer tracings with the smallest peak width at half height are selected for thickness measurements (curve A). 
From curve A and with less preference from curve B, the peak to peak distance can be obtained. To get the total width of the image of the black lipid membrane one dark layer thickness has to be added. This dark layer thickness is taken as equal to the peak-width at half height in situation A. Obviously this corresponds to the smallest width found on the micrographs because any deviation from the orientation in A results in broadening of the peak. In this way we found a peak-to-peak distance of $45 \AA$ (s.D. $=5, n=10$ selected from 6 different sections of two black lipid membranes) and for the dense layers a width of $9 \AA$. No layered pattern could be resolved within the lens-shaped structures throughout a tilting range of $-30^{\circ}$ to $+30^{\circ}$ from the initial specimen orientation.

\section{Discussion}

We have to deal first with the fundamental question of the location of the stabilizing and contrast producing ions in our experiment. The electron microscopical results indicate that binding does occur between the tricomplex ions and the polar layers, because the contrast is produced by ions that increase the local density at the membrane-water interface, where the polar groups of the lipid are located.

Such complex relationships of colloid systems are well known and described in detail by Bungenberg de Jong (1963). In previous articles (Elbers et al., 1965; Elbers \& Ververgaert, 1965) we already paid attention to this work, which provided the initial, theoretical basis of our investigations.

Recent evidence for this theory is supplied by monolayer experiments with egglecithin and dipalmitoyl-lecithin (Shah, 1969). On a subphase of $10^{-3} \mathrm{M}$-uranylacetate a lecithin monolayer tends to solidify with a concomitant increase of the surface potential. It was concluded that $\mathrm{UO}_{2}$ ions interact strongly with the phosphate part of the phosphorylcholine region of the molecule. Pertinent to our observations also is work by White \& Lakshminarayanaiah (1969) who studied association of metal ions with phospholipids as a function of emulsion stability. They found that divalent cations stabilize a lecithin-benzene emulsion in water, whereas CNS ions strongly contribute to this stabilizing action.

Our views originated from a study on tricomplex fixation of phospholipid dispersions (Elbers et al., 1965; Elbers \& Ververgaert, 1965) and are further endorsed by a report on tricomplex fixation of the lung alveolar surfactant mainly consisting of dipalmitoyl-lecithin (Dermer, 1969). In all these investigations the tricomplex reaction allowed visualization of multilayered saturated phospholipid structures without $\mathrm{OsO}_{4}$ treatment.

The fact that in the method described by Henn et al. (1967), the combination of $\mathrm{La}\left(\mathrm{NO}_{3}\right)_{3}$ and $\mathrm{KMnO}_{4}$ is obligatory for successful demonstration of a trilaminar structure could indicate that in this system too, tricomplex fixation is the primary stabilizing factor. In order to test this hypothesis the oxidizing action of the permanganate has to be excluded. Therefore we studied the fixative action of $\mathrm{KMnO}_{4}$ and $\mathrm{La}\left(\mathrm{NO}_{3}\right)_{3}$ on the saturated ditetradecanoylphosphatidylcholine, in which system only ionic interactions are to be expected.

An opalescent ditetradecanoyl-lecithin dispersion in $10^{-2} \mathrm{~N}-\mathrm{La}\left(\mathrm{NO}_{3}\right)_{3}$ flocculates after addition of $\mathrm{KMnO}_{4}\left(10^{-3} \mathrm{M}\right.$ end concentration). Centrifugation and washing yields a purple precipitate. The precipitate has to be considered as a tricomplex according to the criteria mentioned by Elbers et al. (1965). This view is confirmed by electron micrographs showing multilaminar structures with a $40 \AA$ period (Plate II). 

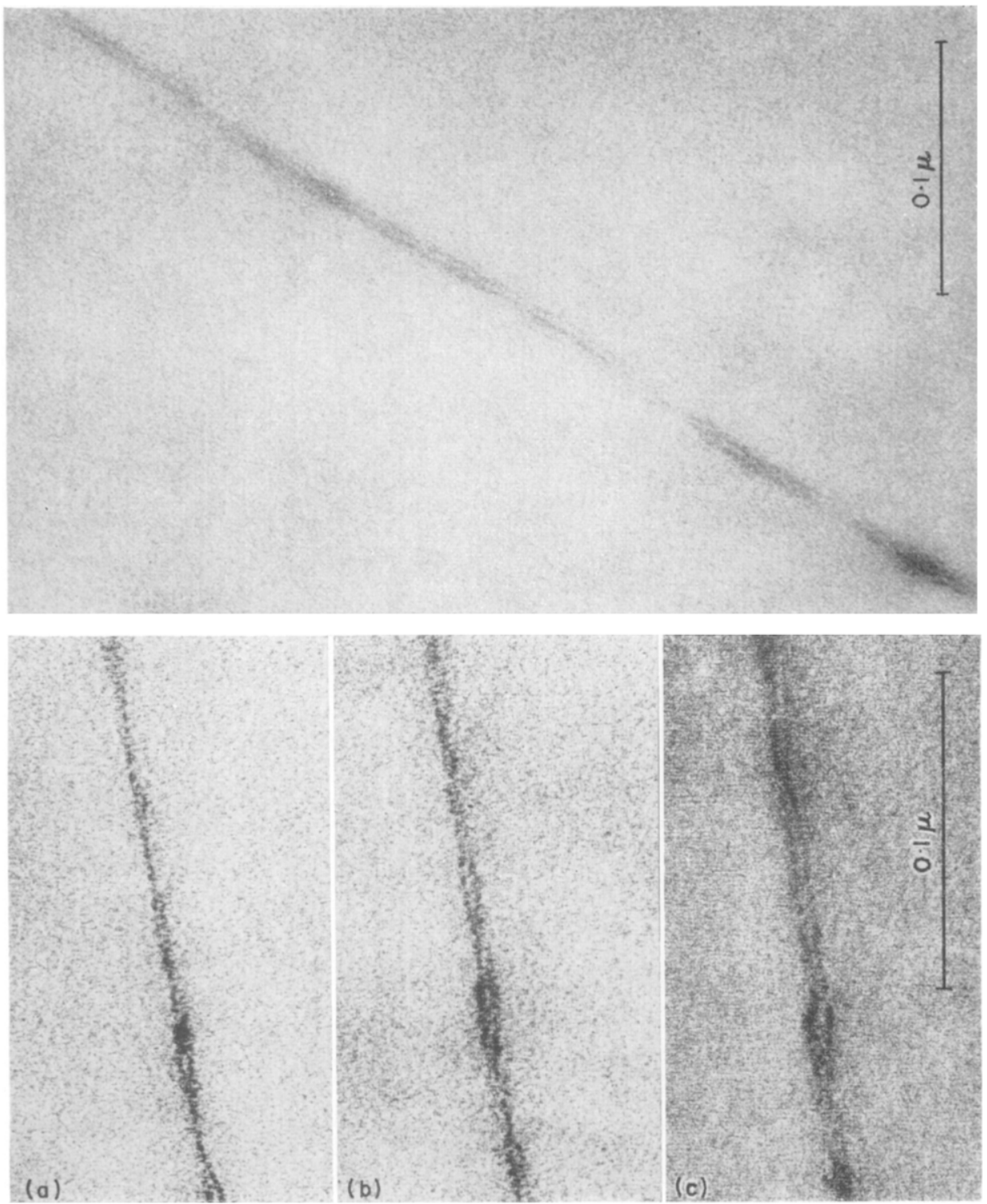

Phate 1. "Top. Crosss-section of black lipid membrane containing egg phosphatidyleholim. and stabilized with $\mathrm{C}_{2}\left(\mathrm{O}_{2}\left(\mathrm{NO}_{3}\right)_{2}\right.$ and $\mathrm{KCNS} . \times 13,000$.

Middle. Higher magnification of the same specimen as in top pranel. The trilaminar pattem is obvious. The discontinuities are probably due to local variations in the thiekness of the speimen. $\therefore 321,000$.

Buttom. Effect of specimen tilting: in a position of the specimen nomal to the electron beam a part of the membrane appears to be single-layered (a). The oorresponding wite of the membanc displays a trilaminar strueture after a tilt of $10^{\circ}$ (b). At an angle of $30^{\circ}$ the triple-layeres pattern is still visible (c). $\% 400,000$. 


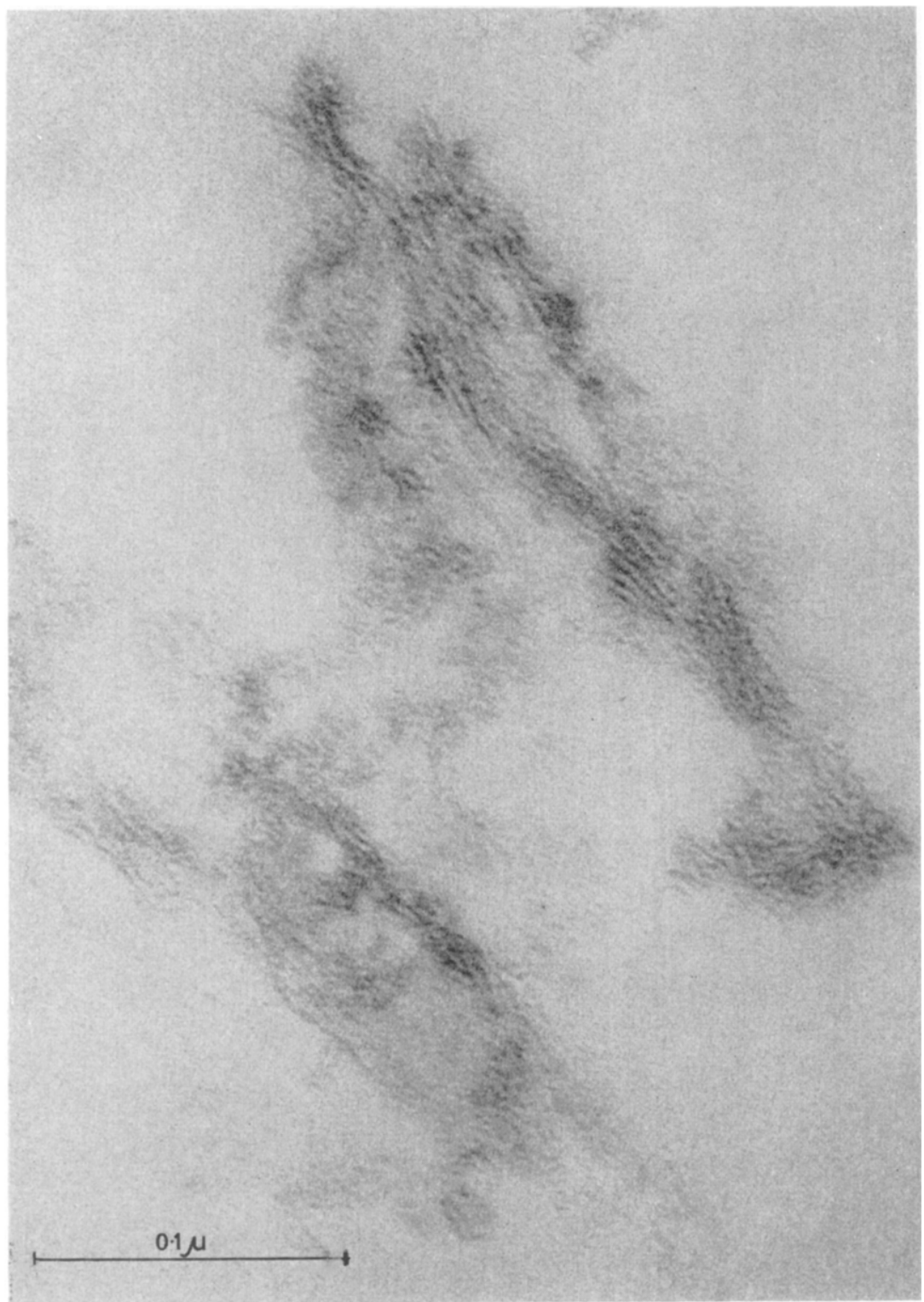

PLATE II. Ditetradecanoyl-lecithin tricomplex with lanthanum and permanganate ions. $\times 375,000$. 
From this experiment, it can be inferred that black lipid membrane stabilization by $\mathrm{KMnO}_{4}$ in the presence of $\mathrm{La}\left(\mathrm{NO}_{3}\right)_{3}$ is partly due to tricomplex formation. Oxidation of the fatty acid part of the phospholipid then probably causes deposition of $\mathrm{MnO}_{2}$ at the black lipid membrane-water interface.

The black film in our experiments is observed as trilaminar, although small deviations from this pattern were also observed so we could frequently discern singlelayered areas within the membrane. By tilting the specimen, however, these structures gradually became trilaminar. Very strikingly, single-layered and trilaminar areas appear to alternate over distances as short as $400 \AA$ so we are led to the conclusion that the film surface exhibits an undulated rather than a smooth pattern. In connection with these orientation experiments we have to revalue the observation (Mueller et al., 1964) that $\mathrm{OsO}_{4}$-fixed brain lipid films do not display triple-layered regions. We think that in most cases an ultrastructural analysis of membranes and in particular of black lipid membranes should be accompanied by tilting experiments. For this purpose, preferably unstained sections should be used because tilting of stained sections could produce a trilaminar image from a single-layered structure according to Haydon (1969).

Another phenomenon is the occurrence along the bilayer of lenses of about $150 \AA$ diameter, occupying not more than $5 \%$ of the total area. The process leading to the formation of these lenses is probably governed by a change of the dielectric constant of the film-solution during thinning. The effect of the dielectric constant of a lecithin solution in benzene-methanol on micelle size has been the subject of an investigation by Elworthy \& McIntosh (1964).

They found that the critical micelle concentration is very low in apolar media (at $\varepsilon=21$; the critical micelle concentration $<2 \times 10^{-3} \mathrm{~g} / \mathrm{ml}$.) whereas a decrease of the dielectric constant from 21 (methanol/benzene $=79 \%$ ) to $2 \cdot 3$ (benzene $=100 \%$ ) involves an increase of the micellar weight from 10,000 to 60,000 . This corresponds to an increase of from 12 to 80 lecithin molecules per micelle. It should be noted that in this range of the dielectric constant, micelles have their apolar part oriented towards the equilibrium solution.

The same relationship between micelle size and $\varepsilon$ presumably will hold for the film solution containing $2 \%$ phosphatidylcholine in methanol, chloroform and hydrocarbon.

This mixture has a lecithin content above the critical micelle concentration corresponding to its dielectric constant $(\varepsilon=9 \cdot 4)$ so large micelles are already present at the start in the lipid solution. During outward diffusion of methanol into the salt solution the dielectric constant of the film solution will be lowered and consequently the micellar size will increase. A final stage is arrived at, in which the micelles are prevented from being removed by border suction through already existing black regions. They form then, together with the two lipid monolayers, lens-like micro. emulsion droplets.

An alternative opinion is held by Andrews \& Haydon (1968). In their view the structureless thick areas found by Henn et al. (1967) represent hydrocarbon compartments within the lipid bilayer, separated off by demixing of the film solution in the process of draining.

Apart from careful considerations on the qualitative results, it has to be emphasized that quantitative data on lipid-layer thickness must be interpreted with even more caution. 
One should keep in mind that the minimal electron imaging dose of $0.1 \mathrm{~A} \mathrm{sec} / \mathrm{cm}^{2}$ will reduce the observed spacing to $75 \%$ of the native value. This has notably been proved for the tricomplex of dioctadecanoyl-lecithin which shows a $69 \AA$ X-ray spacing in contrast to a $51 \AA$ repeat period in electron micrographs (Elbers, 1967).

Applying this figure to the black lipid membrane data we find instead of $45 \pm 5 \AA$ a corrected peak distance of $60 \pm 5 \AA$, and for the total bilayer $69 \pm 5 \AA$. From optical reflection experiments, the thickness of the total egg lecithin bilayer was calculated by Tien (1967) to be $69 \AA$, whereas Cherry \& Chapman (1969), using an average refractive index, found $62 \AA$. Unfortunately we do not know the influence of electron irradiation on the phosphorylcholine part of the phospholipid. We assume that $9 \AA$ as found in the micrographs is the minimum thickness of the total hydrophilic region at the tricomplex membrane-water interface. Subtracting the total width of hydrophilic regions at both sides of the membrane from the total width of the membrane gives us $51 \AA$ as the thickness of the hydrocarbon moiety, which compares favorably with the $48 \AA$ found by Hanai et al. (1964) from capacitance measurements.

A more detailed interpretation of our results can not lie within the scope of this paper, as precise data on the localization of ions in the tricomplex interaction and their influence on the orientation of the polar headgroups of the lipid are not available. Certainly a better understanding of this interaction could be gained by using monolayer techniques.

We are indebted to Mr R. S. M. Revell of the Philips Application Laboratory for his collaboration in the tilting experiments.

We are also grateful to Drs J. B. J. Egberink and R. J. M. Tausk of the Laboratory of Physical Chemistry, Utrecht, for helpful criticism of the manuscript.

\section{REFERENCES}

Andrews, D. M. \& Haydon, D. A. (1968). J. Mol. Biol. 32, 149.

Bungenberg de Jong, H. G. (1963). In Colloid Science II, ed. by H. R. Kruyt. Amsterdam: Elsevier Publishing Company.

Cherry, R. J. \& Chapman, D. (1969). J. Mol. Biol. 40, 19.

Cook, G. M. W., Redwood, W. R., Taylor, A. R. \& Haydon, D. A. (1968). Kolloid Z. $227,28$. Dermer, G. B. (1969). J. Ultrastr. Res. 27, 88.

Elbers, P. F. (1964). In Recent Progress in Surface Science II, ed. by K. G. A. Pankhurst \& A. L. Riddiford. New York: Academic Press.

Elbers, P. F. (1965). Biochim. biophys. Acta, 112, 318.

Elbers, P. F. (1967). Curr. Mod. Biol. 1, 151.

Elbers, P. F. \& Pieters, J. (1964). J. Ultrastr. Res. 11, 25.

Elbers, P. F. \& Ververgaert, P. H. J. Th. (1965). J. Cell Biol. $25,375$.

Elbers, P. F., Ververgaert, P. H. J. Th. \& Demel, R. (1965). J. Cell Biol. 24, 23.

Elworthy, P. H. \& McIntosh, D. S. (1964). Kolloid-Z. u. Z. Polymere, 195, 27.

Hanai, T., Haydon, D. A. \& Taylor, J. L. (1964). Proc. Roy. Soc. A, $281,377$.

Haydon, G. B. (1969). J. Microsc. 90, 1.

Henn, F. A., Decker, G. L., Greenawalt, J. W. \& Thompson, T. E. (1967). J. Mol. Biol. 24, 51.

Korn, E. D. (1966a). Science, 153, 1491.

Korn, E. D. (1966b). Biochim. biophys. Acta, 116, 325.

Moretz, R. C., Akers, C. K. \& Parsons, D. F. (1969). Biochim. biophys. Acta, 193, 1.

Mueller, P., Rudin, D. O., Tien, H. Ti \& Wescott, W. C. (1964). In Recent Progress in Surface Science I, ed. by K. G. A. Pankhurst \& A. C. Riddiford. New York: Academic Press. 
Pangborn, M. C. (1951). J. Biol. Chem. 188, 471.

Riemersma, J. C. (1968). Biochim. biophys. Acta, 152, 718.

Riemersma, J. C. \& Booy, H. I. (1962). J. Histochem. Cytochem. 10, 89.

Robertson, J. D. (1958). Proc. International Conf. Electron Microscopy, Berlin, vol. 2, p. 159.

Shah, D. O. (1969). J. Colloid and Interface Sci. 29, 210.

Stoeckenius, W. (1960). Proc. European Regional Conf. Electron Microscopy, Delft, vol. 2, p. 716.

Stoeckenius, W. \& Mahr, S. C. (1965). Lab. Invest. 14, 458.

Tien, H. Ti (1967). J. Theor. Biol. 16, 97.

Tien, H. Ti (1968). J. Gen. Physiol. 52, 125.

Tien, H. Ti \& Diana, A. L. (1968). Chem. Phys. Lip. $2,55$.

White, M. S. \& Lakshminarayanaiah, N. (1969). Curr. Mod. Biol. 3, 39. 